\title{
Hyperpolarization-Enhanced NMR Spectroscopy with Femtomole Sensitivity Using Quantum Defects in Diamond
}

\author{
Dominik B. Bucher, ${ }^{1,2,8,{ }^{*}}$ David R. Glenn, ${ }^{1}$ Hongkun Park, ${ }^{1,3,4}$ Mikhail D. Lukin, ${ }^{1}$ and Ronald L. Walsworth ${ }^{1,2,4,5,6,7, \dagger}$ \\ ${ }^{1}$ Department of Physics, Harvard University, Cambridge, Massachusetts 02138, USA \\ ${ }^{2}$ Harvard-Smithsonian Centre for Astrophysics, Cambridge, Massachusetts 02138, USA \\ ${ }^{3}$ Department of Chemistry and Chemical Biology, Harvard University, Cambridge, Massachusetts 02138, USA \\ ${ }^{4}$ Center for Brain Science, Harvard University, Cambridge, Massachusetts 02138, USA \\ ${ }^{5}$ Department of Physics, University of Maryland, College Park, Maryland 20742, USA \\ ${ }^{6}$ Department of Electrical and Computer Engineering, University of Maryland, \\ College Park, Maryland 20742, USA \\ ${ }^{7}$ Quantum Technology Center, University of Maryland, College Park, Maryland 20742, USA \\ ${ }^{8}$ Department of Chemistry, Technical University of Munich, 85748 Garching, Germany
}

(Received 3 December 2019; revised manuscript received 17 March 2020; accepted 6 May 2020; published 9 June 2020)

\begin{abstract}
Nuclear magnetic resonance (NMR) spectroscopy is a widely used tool for chemical analysis and molecular structure identification. Because it typically relies on the weak magnetic fields produced by a small thermal nuclear spin polarization, NMR suffers from poor molecule-number sensitivity compared to other analytical techniques. Recently, a new class of NMR sensors based on optically probed nitrogenvacancy (NV) quantum defects in diamond have allowed molecular spectroscopy from sample volumes several orders of magnitude smaller than the most sensitive inductive detectors. To date, however, NV NMR spectrometers have only been able to observe signals from pure, highly concentrated samples. To overcome this limitation, we introduce a technique that combines picoliter-scale NV NMR with fully integrated Overhauser dynamic nuclear polarization to perform high-resolution spectroscopy on a variety of small molecules in dilute solution, with femtomole sensitivity. Our technique advances the state of the art of mass-limited NMR spectroscopy, opening the door to new applications at the picoliter scale in drug and natural-product discovery, catalysis research, and single-cell studies.
\end{abstract}

DOI: $10.1103 /$ PhysRevX.10.021053

\section{INTRODUCTION}

Nuclear magnetic resonance (NMR) sensors based on nitrogen-vacancy (NV) centers, point quantum defects in diamond, provide unprecedented detection of signals from small sample volumes [1-3]. While most early realizations of NV-detected NMR had limited spectral resolution $(\sim 1 \mathrm{kHz})$, recent work has shown that resolution $\sim 1 \mathrm{~Hz}$, sufficient to observe chemical shifts and scalar couplings ( $J$ couplings), can be achieved in micrometer-scale NV

\footnotetext{
*To whom correspondence and requests for materials should be addressed.

dominik.bucher@tum.de

${ }^{\dagger}$ To whom correspondence and requests for materials should be addressed.

walsworth@umd.edu

Published by the American Physical Society under the terms of the Creative Commons Attribution 4.0 International license. Further distribution of this work must maintain attribution to the author(s) and the published article's title, journal citation, and DOI.
}

Subject Areas: Chemical Physics, Physical Chemistry, Quantum Physics
NMR detectors by employing a synchronized readout technique [4-6]. This advance opens the possibility of applying NV NMR to a variety of next-generation analytic technologies, such as single-cell analysis [7] and metabolomics $[8,9]$, and high-throughput screening of masslimited chemical reactions [10-12]. However, because the relevant sample volumes are so small (picoliter scale), NV NMR spectroscopy has to date only been applicable to pure molecular samples $[4,13]$. This restriction precludes many potential chemical, biochemical, and biophysical applications, unless sensitivity improvements can be realized to enable the detection of dilute molecules in solution.

Here, we demonstrate a new technique to address this challenge using high-resolution, micrometer-scale NV NMR in combination with in situ hyperpolarization of the sample nuclear spins, resulting in an improvement of more than 2 orders of magnitude in molecule-number sensitivity for picoliter-scale sample volumes. In particular, we combine NV NMR with Overhauser dynamic nuclear polarization (DNP) [14-16] to transfer the thermal electron-spin polarization of dissolved molecular 
radicals to the nuclei of sample molecules of interest. Integration of the Overhauser DNP system with the NV NMR detector is technically straightforward, because the latter incorporates an efficient GHz-frequency antenna applicable both to NV spin manipulation and to DNP. The combined instrument provides a proton number

(a)
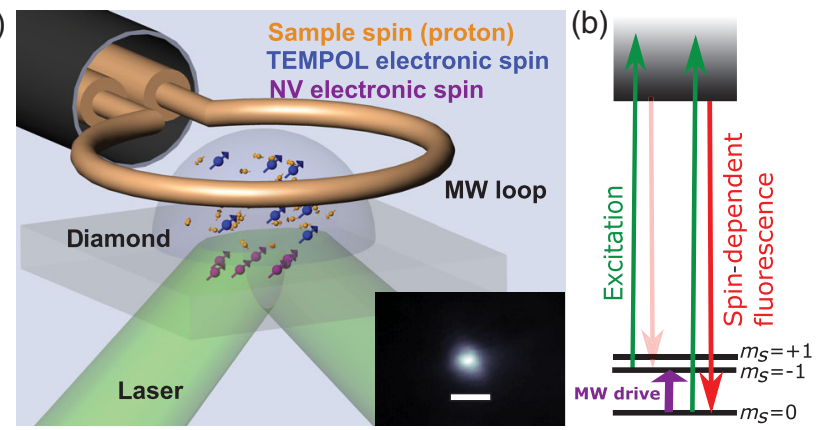

(c)

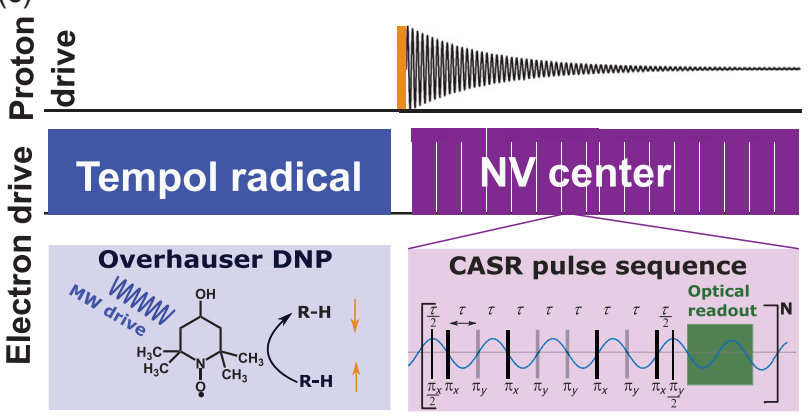

FIG. 1. NV NMR spectroscopy with integrated hyperpolarization. (a) Experimental schematic. Microwave loop antenna near the diamond sensor chip drives both NV (purple) and TEMPOL electronic spins (blue). Hyperpolarized NMR signals from the sample nuclear spins (orange) are detected by NV ensemble fluorescence readout from the diamond chip. Inset: The sensor size is defined by the laser spot size on the diamond (scale bar is $30 \mu \mathrm{m}$ ). (b) The NV center in diamond exhibits spin-dependent fluorescence with triplet ground-state spin transitions that can be manipulated coherently by resonant microwaves (left top). With a suitable MW pulse sequence [CASR, see bottom right of panel (c)] NMR signals can be detected from microscopic volumes and read-out optically. (c) Integrated hyperpolarized NV NMR spectroscopy pulse sequence. In the first half of the pulse sequence ( $\sim 300 \mathrm{~ms})$, the electronic drive is used to hyperpolarize proton spins in the sample via interactions with electronic spins in the TEMPOL radical using Overhauser dynamic nuclear polarization (DNP). In the second half of the pulse sequence ( $\sim 200 \mathrm{~ms})$, a $\pi / 2$ pulse on the protons induces a free nuclear precession (FNP) signal from the hyperpolarized sample, which is detected by NV sensor spins via a coherently averaged synchronized readout (CASR) pulse sequence. Bottom left: Overhauser DNP. Continuous microwave driving saturates the electronic spin transition of the TEMPOL radical. Relaxation leads to a net polarization of proton spins in an organic molecule (R-H), which diffuses relative to the TEMPOL radical. Bottom right: The proton FNP signal is detected by the CASR readout scheme, based on interspersed blocks of identical dynamic decoupling sequences synchronized to an external clock. sensitivity $\sim 10$ picomole $/(\mathrm{Hz})^{1 / 2}$, which enables highresolution NMR spectroscopy from a variety of small molecules in solution at the scale of a single cell, with a sensitivity floor $\sim 50$ femtomole. We note that related techniques based on a combination of microcoil NMR detection with parahydrogen [17] or photochemical hyperpolarization [18] have recently been demonstrated to provide comparable molecule-number sensitivity for microliter sample volumes.

The NV NMR spectrometer consists of a synthetic diamond chip, doped with a high concentration $\left(3 \times 10^{17} \mathrm{~cm}^{-3}\right)$ of NV centers in a thin $(13 \mu \mathrm{m})$ layer at the diamond surface. The spectrometer detects the oscillating magnetic field produced by Larmor precession of the sample nuclear spins, which are weakly coupled via magnetic dipole interactions to the electronic spins of the NV centers. The NV centers are probed using a narrowband dynamical decoupling pulse sequence, leading to an NV spin-state population difference proportional to the oscillating magnetic field amplitude, which is then read out optically using spin-dependent fluorescence [19,20] [Fig. 1(b)].

The active area of the NV ensemble sensor is defined by a focused green laser beam [wavelength $\lambda=532 \mathrm{~nm}$, spot diameter $\sim 20 \mu \mathrm{m}$, see Fig. 1(a) inset] used to initialize and read out the NV electronic spin states. This arrangement results in an effective NMR sensing volume of $\sim 10 \mathrm{pL}$ when a liquid sample is placed in contact with the diamond surface [Fig. 1(a)] [4]. The laser is aligned for total internal reflection within the diamond to reduce the light intensity within the sample. This approach minimizes potential photobleaching, which might otherwise affect the dissolved molecular radicals [21,22]. The diamond is oriented with the [111] axis parallel to the bias magnetic field $\left(B_{0}=84.7 \mathrm{mT}\right)$ provided by a feedback-stabilized electromagnet, and the resulting $\mathrm{NV}$ electron-spin-resonance transitions are driven by a wire loop antenna placed immediately above the diamond surface. Importantly, the bandwidth of this antenna is selected such that it may also be used to drive electron-spin transitions in TEMPOL (4-Hydroxy-TEMPO) molecular radicals dissolved in the liquid sample. Experiments then proceed by applying alternating blocks of (i) Overhauser DNP driving to the dissolved molecular radicals, to transfer thermal electron-spin polarization to nuclear spins of the sample [Fig. 1(c), left]; and (ii) detection of the sample's free nuclear precession (FNP) signal by a coherently averaged synchronized readout (CASR) magnetometry pulse sequence on the NV ensemble sensor [4] [Fig. 1(c), right]. The FNP is induced by applying a $\pi / 2$ pulse on the hyperpolarized nuclear spins of the sample.

\section{RESULTS}

We first perform experiments to test the efficacy of DNP-enhanced NV NMR using a sample of deionized 
(a)

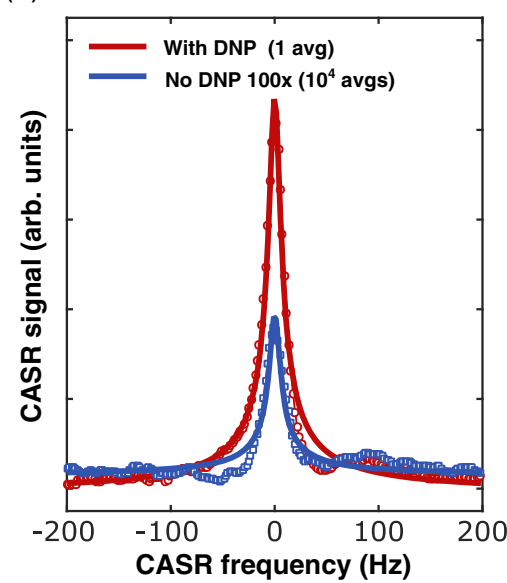

(b)

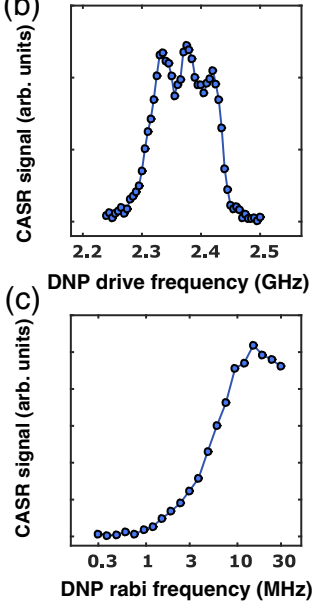

FIG. 2. Hyperpolarization-enhanced NV NMR of water. (a) Comparison of NV NMR spectra obtained from pure water with DNP (red circles, 1 spectrum averaged) and without DNP (blue circles, $10^{4}$ spectra averaged) using a coherently averaged synchronized readout (CASR) pulse sequence. Line shape fits (solid lines) indicate a DNP signal enhancement of 230, with a proton number sensitivity of $10 \mathrm{pmol} / \mathrm{Hz}^{1 / 2}$ for a signal-to-noise ratio (SNR) of 3. (b) Magnitude of CASR-detected signal from a sample of pure water as a function of DNP drive frequency. The triplet structure arises from hyperfine coupling between the electron and ${ }^{14} \mathrm{~N}$ nuclear spins in the TEMPOL radical. (c) Magnitude of CASR-detected signal from a sample of pure water as a function of DNP drive power, expressed in units of the electron Rabi frequency (see methods for details). The maximum DNP signal enhancement is reached at a drive Rabi frequency of approximately $10 \mathrm{MHz}$.

water. TEMPOL radicals are dissolved in the water at a concentration of $20 \mathrm{mM}$, and experiments are carried out using a DNP Rabi frequency of $10 \mathrm{MHz}(0 \mathrm{MHz})$ for the DNP (control) experiment. All other pulse sequence parameters are held constant. Comparison of the CASR-detected NMR spectra shows a $\sim 230 \times$ increase in signal magnitude using DNP compared to the control experiment without DNP, consistent with the expected Overhauser DNP enhancement for TEMPOL at low magnetic field [16,23,24] [Fig. 2(a)]. To achieve this hyperpolarization enhancement of the FNP signal, we optimize polarization transfer from the electronic spins to the sample proton spins by recording the peak CASR-detected FNP signal amplitude while sweeping either the carrier frequency of the DNP drive [Fig. 2(b)] or the DNP Rabi frequency [Fig. 2(c)]. In the first experiment, a triplet structure is visible in the CASR signal enhancement factor due to the ${ }^{14} \mathrm{~N}$ hyperfine splitting of the electronic spin. In the second experiment, a maximum is observed in the CASR signal enhancement at a Rabi frequency of $\sim 10 \mathrm{MHz}$, saturating at higher power likely due to technical issues

associated with sample heating and/or the microwave drive electronics. Addition of TEMPOL radicals to the sample results in a proton spin population lifetime of $T_{1} \approx 150 \mathrm{~ms}$, well matched to the operating linewidth of our NV NMR sensor. Longer sample $T_{1}$ could be achieved by decreasing the TEMPOL concentration, with only a modest reduction in DNP signal enhancement (Supplemental Material, Fig. S1 [25]). For a given initial TEMPOL concentration, the observed NMR signal enhancement remains constant over several days of experiments, indicating no appreciable decrease in concentration due to photobleaching.

We determine the molecule and proton number sensitivity achievable with DNP-enhanced NV NMR spectroscopy in our system [Fig. 3(a)] using samples of tert-butanol $\left[\left(\mathrm{CH}_{3}\right)_{3} \mathrm{COD}\right.$, abbreviated $t$-BuOD $]$ dissolved in heavy water $\left(\mathrm{D}_{2} \mathrm{O}\right)$. The $t$-BuOD proton NMR spectrum is resolved by $3.55 \mathrm{ppm}$ [26] (or $\sim 13 \mathrm{~Hz}$ at $B_{0}=84.7 \mathrm{mT}$ ) from residual semiheavy water (HDO), which occurs in trace quantities in the solvent. By preparing samples with successive dilutions, we observe DNP-enhanced CASR signals from a sample size of $\sim 50$ femtomoles (molecule number, equivalent to a molecule concentration of $5.3 \mathrm{mM}$ in the 10-pL detection volume) with a signal-to-noise ratio (SNR) of 3.5, after $5000 \mathrm{~s}$ of averaging [Fig. 3(b)]. This corresponds to a moleculenumber sensitivity of $3.2 \mathrm{pmol} / \mathrm{Hz}^{1 / 2}$ for $t$-BuOD and a proton number sensitivity of $29 \mathrm{pmol} / \mathrm{Hz}^{1 / 2}$, which is roughly comparable to the observed proton number sensitivity in hyperpolarized water of $10 \mathrm{pmol} / \mathrm{Hz}^{1 / 2}$ [Fig. 2(a)]. We attribute the difference in proton number sensitivity to reduced Overhauser hyperpolarization enhancement in $t$-BuOD compared to water (Table I). In all cases the sensitivity is defined relative to a SNR of 3. The quoted sensitivity includes time taken for both the hyperpolarization and FNP CASR signal detection components of the pulse sequence.

To investigate the generality of the approach, we perform DNP-enhanced NV NMR spectroscopy on a variety of small molecules in solution (all at $0.8-\mathrm{M}$ concentration). Samples of xylene $\left[\left(\mathrm{CH}_{3}\right)_{2} \mathrm{C}_{6} \mathrm{H}_{4}\right]$ dissolved in deuterated dimethyl sulfoxide (DMSO- $d_{6}$ ), dimethylformamide $\left[\left(\mathrm{CH}_{3}\right)_{2} \mathrm{NC}(\mathrm{O}) \mathrm{H}\right]$ dissolved in $\mathrm{D}_{2} \mathrm{O}$, and trimethyl phosphate $\left[\mathrm{PO}\left(\mathrm{OCH}_{3}\right)_{3}\right]$ dissolved in $\mathrm{D}_{2} \mathrm{O}$ were measured with CASR acquisition times of $50 \mathrm{~s}$ [Figs. 4(a)-4(c)]. In each case, molecular NMR spectra were observed with $\mathrm{SNR} \approx 25$; and line-shape fits yielded the expected spectral parameters (line splittings and amplitudes) due to chemical shifts, $J$-coupling interactions, and relative proton abundances. The observed spectral linewidths were on the order of $\Delta f \approx 8-10 \mathrm{~Hz}$ for each measurement. These results are consistent with previously reported spatially inhomogeneous linewidths in our NV NMR 

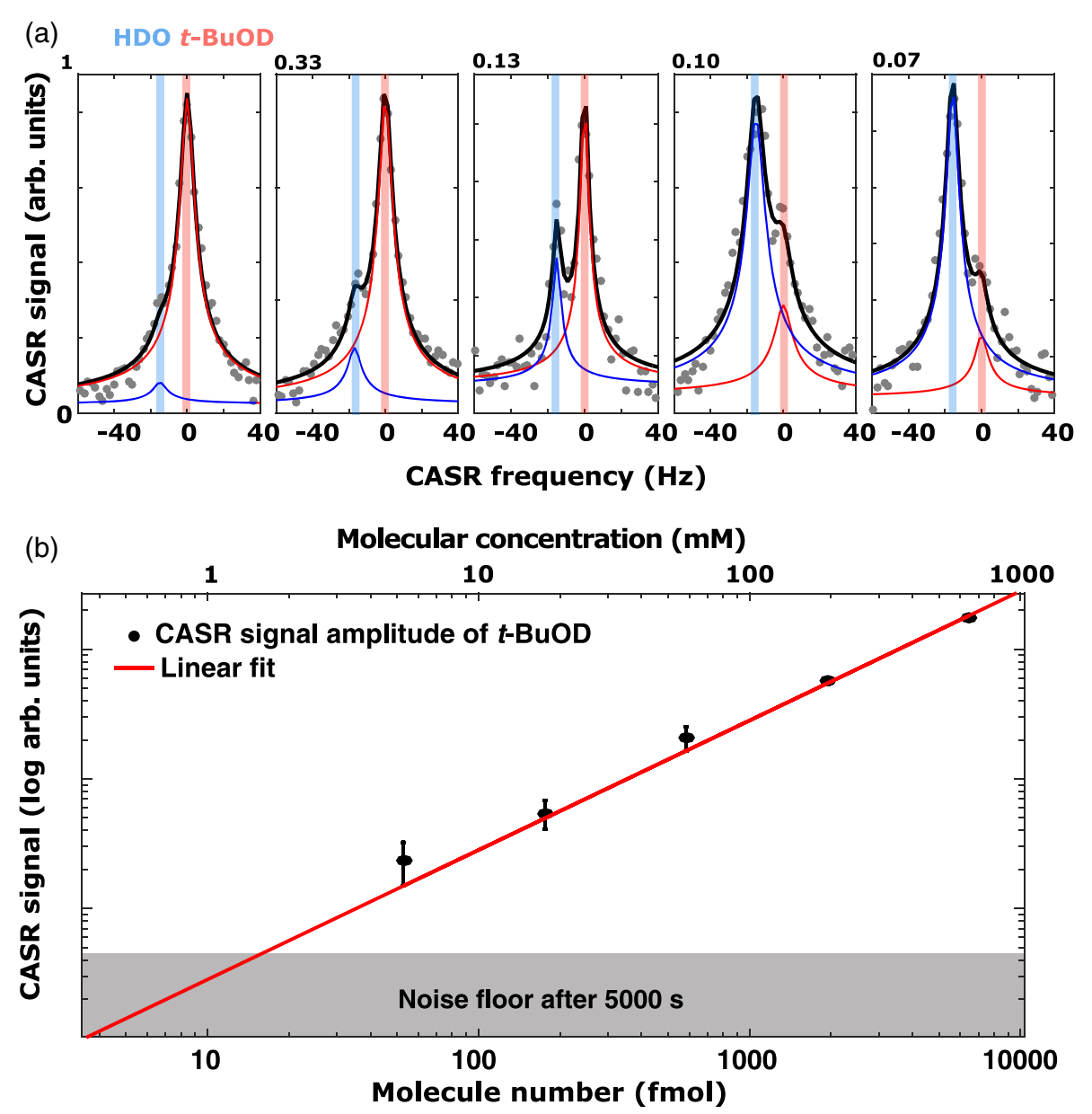

FIG. 3. Sensitivity of hyperpolarization-enhanced NV NMR. (a) CASR-detected spectra of DNP-enhanced $t$-BuOD solutions at different millimolar concentrations $\left(650 \mathrm{mM}, 195 \mathrm{mM}, 58.5 \mathrm{mM}, 17.6 \mathrm{mM}, 5.3 \mathrm{mM}\right.$, from left to right) in $\mathrm{D}_{2} \mathrm{O}$. Spectra are fit to represent the $t$-BuOD sample (red solid line) and residual semiheavy water (HDO) in the $\mathrm{D}_{2} \mathrm{O}$ (blue solid line), with red and blue vertical lines indicating the NMR resonance frequencies of $t$-BuOD and HDO, respectively. (b) Plot of CASR signal fit amplitude of $t$-BuOD against number of sample molecules in the sensing volume (bottom axis) and sample molecular concentration (top axis). Gray area at the bottom marks the noise floor after $5000 \mathrm{~s}$ of averaging with a signal to noise ratio (SNR) $\approx 3.5$ for a concentration of $5.3 \mathrm{mM}$, equivalent to $\sim 50 \mathrm{fmol}$ of sample molecules in the $10 \mathrm{pL}$ detection volume. Error bars represent standard deviation $(1 \sigma)$ of CASR signal measured across three independent experiments.

TABLE I. Summary of observed Overhauser DNP signal enhancements for small organic molecules. Error bars represent standard deviation $(1 \sigma)$ of CASR signals measured across three independent experiments. These molecules cover a broad chemical space, including aromatic protons; aliphatic protons; vinylic protons; protons in functional groups; and protons attached to heteroatoms.

\begin{tabular}{lc}
\hline \hline Sample & Enhancement \\
\hline Water & $230 \pm 8$ \\
Xylene & $82 \pm 7$ \\
Thymine & $84 \pm 3$ \\
DMF & $112 \pm 11$ \\
$t$-BuOD & $126 \pm 5$ \\
Trimethyl phosphate & $118 \pm 7$ \\
\hline \hline
\end{tabular}

spectrometer ascribed to susceptibility-induced broadening [4,27], indicating that introduction of molecular radicals into the samples did not degrade system performance at this level. Finally, we acquired DNP-enhanced NV NMR spectra from a sample of the nucleobase thymine $\left[\mathrm{C}_{5} \mathrm{H}_{6} \mathrm{~N}_{2} \mathrm{O}_{2}\right]$ dissolved in DMSO- $d_{6}$ [Fig. 4(d)]. This measurement required an averaging time of $500 \mathrm{~s}$ to obtain an SNR of 20, largely because of a broadened resonance of the labile $\mathrm{N}-\mathrm{H}$ protons, which we attribute to fast exchange with residual water in the solvent. We determine the DNP enhancement factors for all samples measured in this study to be $\sim 80-230 \times$ (Table I and Supplemental Material, Note 1 [25]), underscoring the broad applicability of our NV NMR technique with femtomole sensitivity. 
(a)

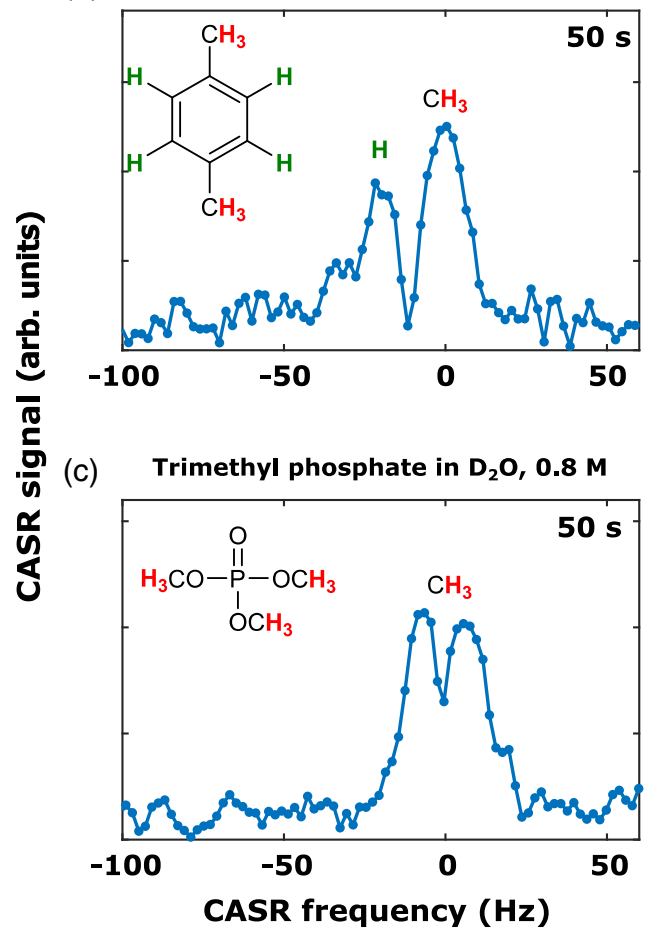

(b) Dimethylformamide in $D_{2} 0,0.8 \mathrm{M}$

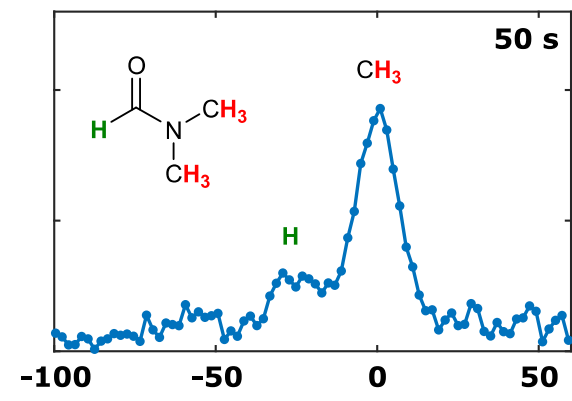

(d) Thymine in DMSO-d6, $0.8 \mathrm{M}$

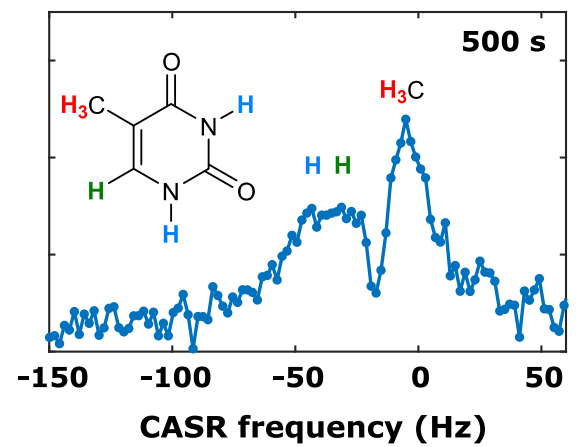

FIG. 4. CASR-detected NMR spectra of hyperpolarized small organic molecules in solution. (a) Xylene in DMSO- $d_{6}$. (b) Dimethyl formamide in $\mathrm{D}_{2} \mathrm{O}$. (c) Trimethyl phosphate in $\mathrm{D}_{2} \mathrm{O}$. (d) Thymine in DMSO- $d_{6}$. All samples were dissolved at a concentration of $0.8 \mathrm{M}$. The features of spectra in (a), (b), and (d) are dominated by chemical shifts, whereas in (c), the $J$ coupling between ${ }^{31} \mathrm{P}$ and ${ }^{1} \mathrm{H}$ splits the $\mathrm{CH}_{3}$ resonances into a doublet. In the thymine spectrum (d), a line broadening effect is observable due to (N-H) proton exchange with small quantities of water dissolved in the DMSO- $d_{6}$. In all spectra, the frequency axis has been set to $0 \mathrm{~Hz}$ for the methyl resonance line.

\section{DISCUSSION}

Overhauser DNP using dissolved molecular radicals is an effective and technically straightforward hyperpolarization method to improve the sensitivity of NV NMR spectroscopy at the micrometer scale by about 2 orders of magnitude. For proton NMR at the macroscopic scale, Overhauser signal enhancement factors typically remain constant for bias magnetic fields up to approximately $\sim 1.5 \mathrm{~T}$ [23]. This suggests that at least one additional order of magnitude of proton NV NMR sensitivity gain (due to linear scaling of the NV NMR signal with thermal spin polarization) is achievable using our technique by increasing the magnetic field from $0.085 \mathrm{~T}$ (reported here) to about $1 \mathrm{~T}$ in future work. Even larger bias fields are likely desirable to increase chemical shift resolution; while driving electron spin resonance transitions at high fields is technically challenging, successful demonstrations of both NV magnetic sensing [13,28] and Overhauser DNP [29,30] have been reported in the literature. Susceptibility mismatches which might limit chemical selectivity at these high fields can be reduced by structural shimming [27]. Further sensitivity improvements may also be possible using advanced NV readout schemes [3].
The extremely small sample volumes accessible with the NV NMR spectrometer may help to mitigate challenges of microwave delivery at high frequencies, due to both (i) reduced dielectric absorption by picoliter volume samples, and (ii) the possibility of using small modevolume resonators to efficiently drive the electronic spins used for Overhauser DNP.

We note that a variety of alternative hyperpolarization schemes have been proposed for NV NMR sensors that take advantage of the optically pumped NV electronic spins themselves as the hyperpolarization source [31-35]. Such schemes, if realized, could provide a streamlined method for high-sensitivity NV NMR that would not rely on dissolved molecular radicals. However, while the achievable polarization of the NV centers is near unity, the small surface-to-volume ratio of the planar diamond chip detector geometry greatly limits the potential effectiveness of such methods in micrometer-scale sample volumes (Supplemental Material, Note 2 [25]). One proposed solution is to instead partially fill the sample volume with NV-doped nanodiamonds, overcoming geometric limitations of the planar diamond surface as a polarization source [31]. In this case, however, the random orientation 
of the individual nanodiamond particles results in a broad angular distribution of $\mathrm{NV}$ quantization axis directions relative to the bias magnetic field, greatly complicating the procedure for polarization transfer [36]. Furthermore, it is often undesirable in practice to apply strong optical pumping to nanodiamonds within the sample volume, due to the possibility for photochemical effects to alter or degrade the sample. For these reasons, we find the introduction of molecular radicals into the sample to be both an effective and practical hyperpolarization technique for NMR signal enhancement at the micrometer scale.

\section{CONCLUSION}

Here, we report an important step toward practical applications of microscale NV NMR. In particular, the ability to measure NMR signals with femtomole sensitivity from picoliter sample volumes may enable new ultrasensitive and high-throughput analytics applications. For example, in drug development and natural-products research, the current state of the art for large-scale screens of binding affinity involves high-throughput nanomolescale synthesis combined with mass spectrometry [10-12]. Introduction of femtomole-scale NV NMR spectroscopy to such a pipeline might simplify sample preparation and provide superior isomeric distinguishability. In the field of metabolomics, the excellent volume selectivity and projected submillimolar-concentration sensitivity of NV NMR may enable quantitative studies at the single-cell level $[37,38]$. Key challenges for future progress include improving chemical sensitivity by operating at tesla-scale magnetic fields; minimizing magnetic susceptibility gradients, particularly at interfaces; and integrating microfluidic sample delivery with the NV NMR instrument. Finally, while the present work has emphasized NV NMR spectroscopy, we note that Overhauser DNP should be equally applicable to magnetic resonance imaging (MRI) techniques. In combination with strong pulsed field gradients [39], NV-detected MRI may enable studies of water diffusion and transport in cells and tissue at the micrometer scale [40].

\section{METHODS}

\section{A. NV ensemble NMR sensor}

The micrometer-scale NMR sensor is based on a ${ }^{12} \mathrm{C}$ enriched (99.999\%) chemical-vapor-deposition diamond chip $(2 \mathrm{~mm} \times 2 \mathrm{~mm} \times 0.5 \mathrm{~mm})$ with a bulk nitrogen $\left({ }^{14} \mathrm{~N}\right)$ concentration of $<8.5 \times 10^{14} \mathrm{~cm}^{-3}$ (Element Six). The diamond is cut so that the lateral faces are perpendicular to [110] and the top face perpendicular to the [100] crystal axis. During the growth process, the chemicalvapor-deposition gas mixture is modified to generate a nitrogen-enriched surface layer of $\sim 13-\mu \mathrm{m}$ thickness, with ${ }^{14} \mathrm{~N}$ density of $\sim 4.8 \times 10^{18} \mathrm{~cm}^{-3}$. Electron irradiation (flux of $1.3 \times 10^{14} \mathrm{~cm}^{-2} \mathrm{~s}^{-1}$ ) for $5 \mathrm{~h}$ and subsequent annealing at $800^{\circ} \mathrm{C}$ in vacuum yields a dense NV ensemble $\left(\sim 3 \times 10^{17} \mathrm{~cm}^{-3}\right)$ in the nitrogen-enriched layer. The $T_{2} *$ of the NV ensemble, measured using a Ramsey pulse sequence, is $\approx 750 \mathrm{~ns}$, while the Hahn echo time $T_{2}$ is $\approx 6.5 \mu \mathrm{s}$. All four diamond edges are polished at a $45^{\circ}$ angle so that the top surface of the diamond is $1 \mathrm{~mm} \times 1 \mathrm{~mm}$. This geometry permits laser excitation of the NV centers using total internal reflection of the incident beam, which reduces light intensity at the sample position above the diamond. In addition, to avoid photobleaching of the TEMPOL radicals we rearrange the sample holder to minimize the laser path through our sample reservoir, compared to the sample holder described in Glenn et al. [4]. The 532-nm laser light is provided by a diode-pumped solid-state laser (Coherent Verdi G7), which is pulsed by an acousto-optic modulator (IntraAction ASM802B47). Each pulse is $5 \mu \mathrm{s}$, where the first microsecond is used to read out the $\mathrm{NV}$ state and the remaining time repolarizes the NV centers. The laser power is around $150 \mathrm{~mW}$, focused down to a spot size of $\sim 20 \mu \mathrm{m}$. The diamond is aligned so that the [111] axis is parallel to the external magnetic bias field $\left(B_{0}\right)$. The ensemble $\mathrm{NV}$ magnetometer has a noise floor of $\sim 20 \mathrm{pT} / \mathrm{Hz}^{1 / 2}$ for ac magnetometry near the proton NMR resonance frequency used in our experiments $(\approx 3.606 \mathrm{MHz})$.

\section{B. Magnetic bias field}

The magnetic bias field $B_{0}=84.7 \mathrm{mT}$ is produced by an electromagnet (Newport Instruments Type A). At this field, the NV resonance frequency $\left(\left|m_{s}=0\right\rangle\right.$ to $\left.\left|m_{s}=-1\right\rangle\right)$ is $\approx 500 \mathrm{MHz}$; the TEMPOL electronic spin resonance frequency is $\approx 2.37 \mathrm{GHz}$; and the proton NMR resonance frequency is $\approx 3.606 \mathrm{MHz}$. The bias field is stabilized with a second NV-diamond magnetometer (feedback sensor) as described in Glenn et al. [4]. For experiments longer than 5 min, slow drifts between the NV NMR experiment and the feedback sensor are corrected every $5 \mathrm{~min}$ by measuring the magnetic field with the CASR sensor using an ESR frequency sweep. The microwave drive for the feedback sensor is delivered by a separate antenna, positioned immediately adjacent to the ESR sensor and driven independently from the main DNP CASR experiment.

\section{DNP CASR pulse sequence parameters}

The full DNP CASR pulse sequence is divided in two parts: (a) the Overhauser hyperpolarization pulse sequence; and (b) FNP detection via a CASR readout sequence. Both are controlled by a programmable pulse generator (Spincore PulseBlaster ESR-PRO, $500 \mathrm{MHz}$ ). The Overhauser microwave (MW) pulse duration is set to $\sim 2 \times \mathrm{NMR} T_{1}$ of the sample (typically around $2 \times 150 \mathrm{~ms}$ ). After the Overhauser sequence, a $\pi / 2$ pulse with a duration of $\approx 150 \mu$ s is applied on the hyperpolarized proton sample to generate the FNP. The FNP signal is then 
read out with the CASR sequence, which is programmed on an arbitrary waveform generator (Tektronix AWG $7122 \mathrm{C}$ ) and triggered by the pulse generator. The full CASR sequence duration is $\sim 4 \times \mathrm{NMR} T_{2}^{*}$ of the sample (typically around $4 \times 50 \mathrm{~ms}$ ). The CASR sequence (for details see Ref. [4]) is based on $X Y 8-6$ subsequences with a duration of $12.45 \mu \mathrm{s}$, chosen to be an integer multiple of (i) the $\mathrm{NV}$ drive period $\left(1 / f_{\mathrm{NV}}=1 / 500 \mathrm{MHz}=2 \mathrm{~ns}\right)$; (ii) the synchronized readout detection period (3320/ $12 \mathrm{~ns}$ ); and (iii) the clock of the waveform generator $\left[1 / f_{\text {clock }}=1 /(12 \mathrm{GHz})\right]$. The $\pi$ and $\pi / 2$ pulse durations used in the $X Y 8-6$ sequences are $\approx 60$ and $30 \mathrm{~ns}$, respectively. Every second pulse sequence is repeated with a $180^{\circ}$ phase shift applied to the last $\pi / 2$ pulse, in order to reject laser and MW noise by subtracting successive pairs of measurements. Thus, one data point of the CASR readout is recorded for two $X Y 8-6$ sequences (i.e., every $24.9 \mu$ s), with a total readout of 8000 points $(199.2 \mathrm{~ms})$. The duration of one full DNP CASR experiment is $\sim 500 \mathrm{~ms}$, which includes the Overhauser sequence and FNP CASR detection.

\section{MW equipment}

Pulse sequences for driving the NV centers (resonance frequency $500 \mathrm{MHz}$ ) are directly synthesized, including both the carrier frequency and the pulse modulation, using an arbitrary waveform generator (Tektronix AWG 7122C), then amplified by a $100 \mathrm{~W}$ amplifier (Minicircuits ZHL $-100 \mathrm{~W}-52+$ ). The Overhauser drive field (resonance frequency $2.37 \mathrm{GHz}$ ) is produced by a signal generator (SRS SG384), pulsed using a microwave switch (Minicircuits ZAWSA-2-50DR+) controlled by the programmable pulse generator, and amplified with a separate 100-W amplifier (ZHL-100W-242+). Both amplified MW drive fields (NV drive and Overhauser drive) are combined using a power combiner (ZACS-242-100W+) and sent to a loop antenna. The antenna is constructed from a $200-\mu \mathrm{m}$ wire, bent into a loop with $\sim 1 \mathrm{~mm}$ diameter, mounted directly on the diamond surface. The loop design is described in detail in Bucher et al. [41] Typically, the sensing volume is located in the center of the loop, corresponding to a distance of $\sim 200-400 \mu \mathrm{m}$ between the sensing volume and the wire. With this configuration, maximum Rabi frequencies are $\sim 30 \mathrm{MHz}$ for driving the NV centers and $\sim 40 \mathrm{MHz}$ for driving the TEMPOL electronic spins. To estimate the TEMPOL Rabi frequency, we perform a Rabi experiment, detected by the NV, on intrinsic electronic spins (dark spins) in the diamond lattice at $g \approx 2$ [42]. The MW power delivery and the DNP enhancement of the sample NMR signal vary somewhat in different experiments due to (i) slightly different antenna orientations upon rebuilding the sensor mount; and (ii) different sample properties (e.g., heat conductance, microwave absorption). We typically use a relatively low NV Rabi frequency of $8.3 \mathrm{MHz}$ for CASR experiments.

\section{E. NMR drive coils}

For applying the $\pi / 2$ pulse on the protons we use a homemade resonant coil at $\approx 3.606 \mathrm{MHz}$ with a quality factor $(Q)$ of $\sim 140$. We typically achieve a proton Rabi frequency of $\sim 4 \mathrm{kHz}$ by driving this coil using our signal source (Rigol DG 1032) without amplification.

\section{F. Data analysis}

Each FNP signal measurement gives a time-series dataset consisting of 8000 data points from the CASR sequence. The first 20 data points $(\approx 0.5 \mathrm{~ms})$ are discarded because of artifacts (e.g., coil ringdown) associated with the $\pi / 2$ pulse on the proton spins. For experiments in which signal averaging is required, the averaging is performed in the time domain, before data are mean subtracted. The time-series data are zero padded to a length of 20000 points (498 ms), corresponding to the full duration of the combined pulse sequence (CASR + Overhauser). In addition, we filter the datasets by multiplying by an exponential filter function $e^{-t / \tau_{f}}$ with a time constant $\tau_{f}$. We use a time constant $\tau_{f}=50 \mathrm{~ms}$ for the data shown in Fig. 2(a), and $\tau_{f}=250 \mathrm{~ms}$ for the data in Figs. 3(a), 3(b), and 4. After averaging and filtering, the time-series datasets are then Fourier transformed in MATLAB. In all plots, we show the absolute value of the Fourier transformation (CASR signal). Each experiment was performed at least three times to verify reproducibility (see Supplemental Material, Fig. S2 [25]). In most cases, the variability of the measured signal size is dominated by antenna alignment or sample concentration uncertainties, rather than the intrinsic sensitivity of the CASR readout.

The dataset of Fig. 3(a) is fit to the sum of two modified Lorentzian functions:

$$
\begin{aligned}
F(f)= & \frac{A_{t-\mathrm{BuOD}} L_{t-\mathrm{BuOD}}}{\sqrt{\left[\left(f-f_{t-\mathrm{BuOD}}\right)^{2}+L_{t-\mathrm{BuOD}}{ }^{2}\right]}} \\
& +\frac{A_{\mathrm{HDO}} L_{\mathrm{HDO}}}{\sqrt{\left[\left(f-f_{\mathrm{HDO}}\right)^{2}+L_{\mathrm{HDO}}{ }^{2}\right]}} .
\end{aligned}
$$

Here, $A$ is the amplitude, $L$ the linewidth, and $f$ the resonance frequency for $t$-BuOD and HDO, respectively. (Note that the $L$ as defined here is broader by a factor of $\sqrt{3}$ compared to the power spectrum of the CASR signal, which was plotted in our previous paper [4].) The amplitude $A_{t-\mathrm{BuOD}}$ of the $t$-BuOD component of the spectrum is plotted in Fig. 3(b). In Figs. 2(b) and 2(c) we plot the amplitude of the CASR signal against the swept experimental parameter. All amplitudes are normalized to a synthetic magnetic ac signal, generated by an external loop antenna positioned near to the diamond sensor chip, with the ac field set to have 100-pT amplitude near the proton Larmor frequency $(\approx 3.6 \mathrm{MHz})$. In all cases we define the sensitivity as well as the proton number limit of detection 
for a signal-to-noise ratio (SNR) of 3. We computed the SNR by dividing the signal amplitude (maximum of the signal) by the standard deviation of a spectral region without a signal.

The DNP enhancement factors for water were determined directly by comparing the measured CASR signal amplitudes with DNP drive on and off (Fig. 2). The enhancement factors for dilute small molecules could not be measured in this manner, since the signals were too small to detect without Overhauser DNP. In these cases, we (i) referenced the measured CASR signal amplitudes with DNP drive on to the synthetic ac signal; (ii) compared these measurements to the water CASR signal amplitudes with DNP drive on and off; and then (iii) used the known molecular concentration to determine the DNP enhancement factor.

\section{G. Samples}

As a hyperpolarizing agent, we used TEMPOL from Sigma Aldrich (catalog no. 176141) without further modification. The samples $p$-xylene, trimethyl phosphate, $N, N$ dimethylformamide, thymine, and tert-butanol were purchased from Sigma Aldrich (catalog no. 296333, no. 241024, no. D4551, no. T0376, and no. 471712). The deuterated samples $\mathrm{D}_{2} \mathrm{O}$ and dimethyl sulfoxide- $d 6$ (DMSO- $d 6$ ) were obtained from Cambridge Isotope Laboratories, Inc. (catalog no. DLM-4-100 and no. DLM-10-10). In the data of Fig. 3(a) we observe an HDO resonance line that is caused by (i) residual water in the purchased sample, and (ii) water vapor absorption during handling in the laboratory. Diluted samples were prepared by either weighing the sample or using microliter pipettes. Solvents and samples were not degassed in any of the experiments.

\section{ACKNOWLEDGMENTS}

We thank Matthew Rosen for initial help with the Overhauser polarization scheme. This material is based on work supported by, or in part by, the U.S. Army Research Laboratory and the U.S. Army Research Office under Contract/Grant No. W911NF1510548; as well as the Moore Foundation. D. B. B. was partially supported by the German Research Foundation (BU 3257/1-1).

The authors declare no competing interests.

D. B. B., D. R. G., and R. L. W. invented the Overhauser enhanced CASR NV-diamond spectroscopy technique. D. B. B., D. R. G., and R. L. W. designed the experiments and analyzed the data. D. B. B. modified the NV NMR spectrometer for hyperpolarization and carried out the experiments. M. D. L, H. P., and R. L. W. conceived the application of NV diamond magnetometry to NMR detection at short length scales. All authors discussed the results and participated in writing the manuscript.
[1] H. J. Mamin, M. Kim, M. H. Sherwood, C. T. Rettner, K. Ohno, D. D. Awschalom, and D. Rugar, Nanoscale Nuclear Magnetic Resonance with a Nitrogen-Vacancy Spin Sensor, Science 339, 557 (2013).

[2] T. Staudacher, F. Shi, S. Pezzagna, J. Meijer, J. Du, C. A. Meriles, F. Reinhard, and J. Wrachtrup, Nuclear Magnetic Resonance Spectroscopy on a (5-Nanometer) ${ }^{3}$ Sample Volume, Science 339, 561 (2013).

[3] I. Lovchinsky et al., Nuclear Magnetic Resonance Detection and Spectroscopy of Single Proteins Using Quantum Logic, Science 351, 836 (2016).

[4] D. R. Glenn, D. B. Bucher, J. Lee, M. D. Lukin, H. Park, and R. L. Walsworth, High-Resolution Magnetic Resonance Spectroscopy Using a Solid-State Spin Sensor, Nature (London) 555, 351 (2018).

[5] J. M. Boss, K. S. Cujia, J. Zopes, and C. L. Degen, Quantum Sensing with Arbitrary Frequency Resolution, Science 356, 837 (2017).

[6] S. Schmitt et al., Submillihertz Magnetic Spectroscopy Performed with a Nanoscale Quantum Sensor, Science 356, 832 (2017).

[7] A. Marusyk, V. Almendro, and K. Polyak, Intra-Tumour Heterogeneity: A Looking Glass for Cancer?, Nat. Rev. Cancer 12, 323 (2012).

[8] M. Fessenden, Metabolomics: Small Molecules, Single Cells, Nature (London) 540, 153 (2016).

[9] J. L. Griffin and J.P. Shockcor, Metabolic Profiles of Cancer Cells, Nat. Rev. Cancer 4, 551 (2004).

[10] N. J. Gesmundo, B. Sauvagnat, P. J. Curran, M. P. Richards, C. L. Andrews, P. J. Dandliker, and T. Cernak, Nanoscale Synthesis and Affinity Ranking, Nature (London) 557, 228 (2018).

[11] A. B. Santanilla et al. Nanomole-Scale High-Throughput Chemistry for the Synthesis of Complex Molecules, Science 347, 49 (2015).

[12] S. Lin et al., Mapping the Dark Space of Chemical Reactions with Extended Nanomole Synthesis and MALDI-TOF MS, Science 361, eaar6236 (2018).

[13] N. Aslam et al., Nanoscale Nuclear Magnetic Resonance with Chemical Resolution, Science 357, 67 (2017).

[14] A. W. Overhauser, Polarization of Nuclei in Metals, Phys. Rev. 92, 411 (1953).

[15] T. R. Carver and C. P. Slichter, Experimental Verification of the Overhauser Nuclear Polarization Effect, Phys. Rev. 102, 975 (1956).

[16] E. Ravera, C. Luchinat, and G. Parigi, Basic Facts and Perspectives of Overhauser DNP NMR, J. Magn. Reson. 264, 78 (2016).

[17] J. Eills, W. Hale, M. Sharma, M. Rossetto, M. H. Levitt, and M. Utz, High-Resolution Nuclear Magnetic Resonance Spectroscopy with Picomole Sensitivity by Hyperpolarization on a Chip, J. Am. Chem. Soc. 141, 9955 (2019).

[18] M. Mompeán, R. M. Sánchez-Donoso, A. de la Hoz, V. Saggiomo, A. H. Velders, and M. V. Gomez, Pushing Nuclear Magnetic Resonance Sensitivity Limits with Microfluidics and Photo-Chemically Induced Dynamic Nuclear Polarization, Nat. Commun. 9, 108 (2018).

[19] M. W. Doherty, N. B. Manson, P. Delaney, F. Jelezko, J. Wrachtrup, and L. C. L. Hollenberg, The Nitrogen-Vacancy Colour Centre in Diamond, Phys. Rep. 528, 1 (2013). 
[20] E. Abe and K. Sasaki, Tutorial: Magnetic Resonance with Nitrogen-Vacancy Centers in Diamond-Microwave Engineering, Materials Science, and Magnetometry, J. Appl. Phys. 123, 161101 (2018).

[21] L. Schlipf et al., A Molecular Quantum Spin Network Controlled by a Single Qubit, Sci. Adv. 3, e1701116 (2017).

[22] F. Shi et al., Single-Protein Spin Resonance Spectroscopy under Ambient Conditions, Science 347, 1135 (2015).

[23] J. H. Lee, Y. Okuno, and S. Cavagnero, Sensitivity Enhancement in Solution NMR: Emerging Ideas and New Frontiers, J. Magn. Reson. 241, 18 (2014).

[24] C. Griesinger, M. Bennati, H. M. Vieth, C. Luchinat, G. Parigi, P. Höfer, F. Engelke, S. J. Glaser, V. Denysenkov, and T. F. Prisner, Dynamic Nuclear Polarization at High Magnetic Fields in Liquids, Prog. Nucl. Magn. Reson. Spectrosc. 64, 4 (2012).

[25] See Supplemental Material at http://link.aps.org/ supplemental/10.1103/PhysRevX.10.021053 for detailed information.

[26] H. E. Gottlieb, V. Kotlyar, and A. Nudelman, NMR Chemical Shifts of Common Laboratory Solvents as Trace Impurities, J. Organic Chem. 62, 7512 (1997).

[27] H. Ryan, A. Smith, and M. Utz, Structural Shimming for High-Resolution Nuclear Magnetic Resonance Spectroscopy in Lab-on-a-Chip Devices, Lab Chip 14, 1678 (2014).

[28] V. Stepanov, F. H. Cho, C. Abeywardana, and S. Takahashi, High-Frequency and High-Field Optically Detected Magnetic Resonance of Nitrogen-Vacancy Centers in Diamond, Appl. Phys. Lett. 106, 063111 (2015).

[29] G. Liu, M. Levien, N. Karschin, G. Parigi, C. Luchinat, and M. Bennati, One-Thousand-Fold Enhancement of High Field Liquid Nuclear Magnetic Resonance Signals at Room Temperature, Nat. Chem. 9, 676 (2017).

[30] E. V. Kryukov, K. J. Pike, T. K. Y. Tam, M. E. Newton, M. E. Smith, and R. Dupree, Determination of the Temperature Dependence of the Dynamic Nuclear Polarisation Enhancement of Water Protons at 3.4 Tesla, Phys. Chem. Chem. Phys. 13, 4372 (2011).

[31] Q. Chen, Resonance-Inclined Optical Nuclear Spin Polarization of Liquids in Diamond Structures, Phys. Rev. B 93, 060408 (2016).
[32] D. Abrams, M. E. Trusheim, D. R. Englund, M. D. Shattuck, and C. A. Meriles, Dynamic Nuclear Spin Polarization of Liquids and Gases in Contact with Nanostructured Diamond, Nano Lett. 14, 2471 (2014).

[33] D. A. Broadway, J.-P. Tetienne, A. Stacey, J. D. A. Wood, D. A. Simpson, L. T. Hall, and L. C. L. Hollenberg, Quantum Probe Hyperpolarisation of Molecular Nuclear Spins, Nat. Commun. 9, 1246 (2018).

[34] P. Fernández-Acebal et al., Toward Hyperpolarization of Oil Molecules via Single Nitrogen Vacancy Centers in Diamond, Nano Lett. 18, 1882 (2018).

[35] F. Shagieva, S. Zaiser, P. Neumann, D. B. R. Dasari, R. Stöhr, A. Denisenko, R. Reuter, C. A. Meriles, and J. Wrachtrup, Microwave-Assisted Cross-Polarization of Nuclear Spin Ensembles from Optically Pumped NitrogenVacancy Centers in Diamond, Nano Lett. 18, 3731 (2018).

[36] Q. Chen, I. Schwarz, F. Jelezko, A. Retzker, and M. B. Plenio, Optical Hyperpolarization of 13 C Nuclear Spins in Nanodiamond Ensembles, Phys. Rev. B 92, 184420 (2015).

[37] S. Jeong, R. Eskandari, S. M. Park, J. Alvarez, S. S. Tee, R. Weissleder, M. G. Kharas, H. Lee, and K. R. Keshari Real-Time Quantitative Analysis of Metabolic Flux in Live Cells Using a Hyperpolarized Micromagnetic Resonance Spectrometer, Sci. Adv. 3, e1700341 (2017).

[38] J. L. Markley, R. Brüschweiler, A. S. Edison, H. R. Eghbalnia, R. Powers, D. Raftery, and D. S. Wishart, The Future of NMR-Based Metabolomics, Curr. Opin. Biotechnol. 43, 34 (2017).

[39] K. Arai, C. Belthangady, H. Zhang, N. Bar-Gill, S. J. DeVience, P. Cappellaro, A. Yacoby, and R. L. Walsworth, Fourier Magnetic Imaging with Nanoscale Resolution and Compressed Sensing Speed-Up Using Electronic Spins in Diamond, Nat. Nanotechnol. 10, 859 (2015).

[40] D. S. Novikov, E. Fieremans, S. N. Jespersen, and V. G. Kiselev, Quantifying Brain Microstructure with Diffusion MRI: Theory and Parameter Estimation, arXiv:1612.02059.

[41] D. B. Bucher et al., Quantum Diamond Spectrometer for Nanoscale NMR and ESR Spectroscopy, Nat. Protoc. 14, 2707 (2019).

[42] E. Bauch, C. A. Hart, J. M. Schloss, M. J. Turner, J. F. Barry, P. Kehayias, S. Singh, and R. L. Walsworth, Ultralong Dephasing Times in Solid-State Spin Ensembles via Quantum Control, Phys. Rev. X 8, 031025 (2018). 